\title{
Logic, Reasoning, Decision-Making
}

\author{
Nataliia Reva \\ Ph.D. student of the Taras Shevchenko National University of Kyiv \\ (Kyiv, Ukraine) \\ E-mail: natalie.reva@gmail.com \\ ORCID: 0000-0002-3931-3755
}

The author focuses the main attention on the people's everyday reasoning and its mechanism developing the idea that the human mind influenced by the bounded rationality does not always use the logical tools to make a decision. More precisely, the author is talking about the deductive reasoning cherished by Aristotle and its place in real-life reasoning. The main questions raised in this paper are: (1) Does Aristotelian syllogism work for real life? (2) Are we the rational creatures with the "computer mind"? (3) What differs us from the Artificial Intelligence in the decision-making process? Comparing the processing work of a computer program, especially the Artificial Intelligence, with the everyday reasoning of the human mind, the author comes to the conclusion that Aristotelian logic has a more significant impact on the development of the AI than on human thinking. One of the main reasons is that the machines, no matter how advanced they are, are free from the external influences. They use the program put in their mainboard without "thinking" of the outcomes. They do not care if the results of their calculations or actions hurt someone. For example, those new autonomous cars with the movement detectors enable the driver to sleep during the trip, because the machine is driving for you, steering wheel angle, checking the road conditions and correcting the path itself. At the same time, it will not think of your body position and will you be hurt or not in case of the abrupt stop when the child appears in front of the car. It does as it was programed (or how it had learn) to do. While human can use different tools to reach their goal and make the decisions in real life. People do not stay rational all the time and, unlike the machines, can use intuition or do some moves without thinking just by force of habit. These unconscious forces can both help and hurt human decisions.

Keywords: logic, syllogism, deductive reasoning, bounded rationality, decision-making, Artificial Intelligence

Received: September 13, 2018; accepted: October 3, 2018

Future Human Image, Volume 10, 2018: 76-84.

DOI: $10.29202 / \mathrm{fhi} / 10 / 8$

(C) Reva, Nataliia, 2018 


\section{God save logic}

When we ask anyone, except the logicians, what logic is and what it serves for, the answer will be that the logic is a rational way of thinking. This correlation between rationality and logic is unbreakable in our head. For instance, when somebody claims to be a vegetarian, but continue to eat chicken saying that the birds are stupid, we may notice that his/her arguments lack logic. For us, it may sound unreasonable to eat chicken due to its low intelligence. At the same time, if we ask the same question to the logician, he will explain to you that logic is a science of proper reasoning. To say more generally, it is the systematic research of the form of valid inference, which is represented by a specific logical relation between the premises and its conclusion. Therefore, if our vegetarian friend continues to justify his/her decision by saying that due to the low intelligence the chickens fail to realize of what is happening before they die, thus, they die without any pain enable to capture that fatal moment. These given arguments almost every time act positively on us. We start to find our friend's position plausible. This is the pure magic of the arguments.

We live in the society that forces us to justify every decision, idea or even dream we have. This tradition of "being rational" came to us from Ancient Greece. In particular Aristotle's incomparable influence on the development of Western thought. This Greek philosopher created what we call today the argument. Aristotle's logic ground on one notion - the syllogism - that is a kind of logical argument used in the deductive reasoning. Recalling our previous example about the chicken, we can write it down in the following structure:

1. Our vegetarian friend does not eat the meat of animals who die in suffering.

2. Chicken does not suffer when it is dying.

3. Therefore, our friend eats chicken.

This is the typical example of Aristotle's syllogism that has two premises and a conclusion. In fact, the syllogism is nothing else as the deduction. Deductive reasoning always involves the logical consequence of given premises. Accordingly, if all premises are true, the conclusion is necessarily true. That is why we accept our friend's explanation, even if we are not sure does chicken suffer for real or not. Sure, we can try to get into the chicken head, test the neural impulses in their brain in ordinary life and the situation of danger, yet, we cannot be sure that our interpretation of the results will reveal their real state. We can only guess. Still, we cannot feel for them.

Aristotle thought of that too. Thus, he classified the proofs into two categories: "perfect" or "complete" deductions and "imperfect" or "incomplete" deductions. In Organon, to be more precise in the Prior Analytics, the philosopher described the complete deduction as the one that needs no external term to show the necessary result [Aristotle, 1889: 24b23-24]. Hence, the incomplete deduction needs the additional justification. As follows, we may compare the complete deductions to some kind of the axioms as in the mathematics. Since they do not need the third-party assistance to stay true or to prove they are true, they can stand for the basis for any other deductions that are incomplete. Thereby, to determine the incomplete deduction Aristotle insists that we should reduce it to the universal deduction for support. Nevertheless, how often do you search for this "perfect base" in your normal life before you make a decision or accept someone's idea?

We take almost every issue considering that we have the full knowledge of it (or at least all that we need for the conclusion). We do not verify every statement we hear or check a thousand of possibilities before choosing one toothbrush. We do not make these hard and 
unnecessary combinations in our head telling ourselves that this toothbrush belongs to the kind of toothbrushes and as all the toothbrushes are used to brush the teeth this one has the same mission as every other toothbrush. To be honest, we do not even need to deduct this. We just know what the toothbrush is made for when we see it. Of course, there are some unusual situations when we can apply the toothbrush differently, like the "punishment" in the army, for instance, when a soldier must polish the floor or a toilet with it. However, it is an unconventional application, so ordinarily; it does not instantly come to our mind. Still, everyone agrees that the usage of the toothbrush is not the universal knowledge.

Certainly, we can follow Aristotle's illustration and even accept the division of knowledge he proposes (discursive knowledge is made through argument and non-discursive, that is the knowledge of the first principles). In simple terms, there is the knowledge we get from our "ratio" by reflection, and the one directed by our intuition that Aristotle claims to be "immediate parcel" delivered from our mind (nous) without any arguments. To which class would you rank our knowledge about the toothbrush? We certainly do not waste our time to verify every toothbrush on its "toothbrushness." It could be the intuitive knowledge in sporadic cases, like when the aborigines from some "mumbo jumbo" tribe instantly figure out themselves what for is this "stick with the hairs." In the same way, the men who know the applying of the toothbrush can intuitively decide is it the new or the old one, was it used to brush the teeth or to clean the sneaker sole, etc.

For Aristotle, intuition is the most fundamental level of operation because it provides us with the first principles, like the primary principles and concepts, the universal propositions, the laws of logic, and so forth. We cannot doubt the intuition because everything: science, philosophy, knowledge, logic, etc. — is built upon this universal foundation. However, does really the first impressions that seem to come from our feelings and hunches that we call the intuition are not just the quick work of our brain that is hard for us to be captured? Perhaps, the instantaneous discernment that appears in our mind is nothing else that the product given by the processing machine in our head. In this case, the immediate comprehension we get is the same discursive knowledge. The main difference is that it was made unconsciously.

Much better for logic. Since our brain works as the computer, we can always try to formalize and compile its impulses into a system. We need nothing but to penetrate into the subconscious level and check the data processing process. For now, we can only assume that this innate, automatic reasoning performs in the same manner as the rational thinking. Nevertheless, the question arises: why people still make some illogical decisions or do some irrational deeds? Perhaps an error is laid in our process of comprehension the inside information submitted by our mind. To put it simply, we overthink the problem. On the other hand, the obstacle may come from outside: the bad weather, rude client, headache or on the contrary your favorite music, new commercial, familiar face, etc. There are millions of inciting factors that may intrude our thinking process and influence (or even change) our decision, vision, and position. Unfortunately, for Aristotle, we do not always use the deductive reasoning, construct the strong arguments pro and contra, and verify every tiny possibility. People are lazy creatures. So, we always tend to simplify our life in any of its manifestations. The reasoning is not an exception. 


\section{Repetitive thinking}

American psychologist Gary Klein, who had been studied the practice of decision-making in the extreme situations, concluded that the "do-or-die" decisions are almost always made intuitively on the basis of unconscious or dimly perceived impressions. In his Sources of Power: How People Make Decisions Klein emphasizes that the most important role in this instant reaction is played by experience and the implicit knowledge obtained before. The "source of power" involved in the process of making fast and good decisions in the stressful situations of high importance or danger risks is not the analytical one. These are the mental stimulus, which helps us to size quickly the circumstances we are in and push us to act [Klein, 1999: 84].

Sometimes we even deliberately accustom ourselves to some actions to be able to repeat them right away. As an example, a woman may practice some self-defense techniques like Krav Maga to be sure that she will protect herself if someone attacks her. At times of danger like in the previous case, we often do not have time to study the situation and logically deduce the most suitable option for action. The time pressure demands the effective rapid moves. Besides, the everyday practice do not only concern fighting, dancing or other external movements. It also may be about getting used to fast thinking. Let us look at another case. Tesla in his autobiography My Inventions confessed that his father forced him and his brother to count the large numbers in mind every evening before eating. As he said himself, this in former times annoying exercise soon become a handy habit that helped a lot this genius physicist [Tesla, 1919]. Now you see that training mind may be as useful as training muscles.

Habits appear quickly. One morning you woke up and took your phone to see the time, then you repeat this action the next day, and the day after, and now you are doing it every day without realizing that this move became your routine. Therefore, when something interrupts this cycle, you unconsciously assume that something goes wrong. I know that this illustration with the phone is a little bit exaggerating, but take a look at the example Klein gives us. A good firefighter knows the signs of fire, its sound, its smell and so on. Thus, when he does not see or hear these features, he should become more alert. Once a team of firefighters miraculously escaped the death, leaving the house a minute before its roof collapsed. When the journalist asked their leader, he did not know what had directed him to the exit. Later talking to Klein, he realized that the house was quieter than it should be. This simple detail alarmed him and gave him a clue of what he needs to do next [Klein, 1999: 33]. Does it mean that our mind works the same way as a registered computer program?

Shifting our attention from the decisions we make under pressure, we have plenty of other situations we should make up our mind with. As I said, the majority of people are lazy and neglectful in their nature. So, in ordinary life we choose the first yogurt we like the appearance of; we sit on the seat that we liked for the first sight even if the bus is empty and this seat is at the end of the car; we decide to switch the radio channel on the first chords of the song; and so on. We do these actions without thinking like if some force that we do not control pushes us. This push, gentle as it is, may be produced by our previous experience. We could learn about the yogurt from the TV advertising; read in an article that people who were sitting in the back seats were the only ones who did not get hurt in some bus accident; have the bad memories with a similar melody; etc.

The same phenomenon happens when we fall in love. We do not calculate all the good and bad qualities of our partner we just feel the attraction created by the pheromones, lovely 
smells or nice colors our soulmate prefers. We start to analyze the situation only when the veil of the first intense feelings fall. Therefore, our laziness is not at all a bad characteristic; we just economize our energy for the most useful things. Just imagine how long it would take to pick one yogurt if we study all offered products in the mall. It would take a lot of time and energy that we could spend on something more useful and intellectual. In Descartes' Error written by Antonio Damasio, the author illustrates the case of the patient with ventromedial prefrontal damage. This poor man could not select the date for his next appointment with the doctor (between two alternatives) without a half-hour enumeration of all reasons for and against for each of the two dates. The writer is sure that this kind of behavior is a good illustration of the "limits of pure reason" and the "calamitous consequence of not having automated mechanisms of decision making" [Damasio, 1995: 194].

Returning to our talk about the practice and its fetuses let me show you the other case I have found in Malcolm Gladwell's book Blink. The Power of Thinking without Thinking. When we talk about the theater of improvisation, the thing that amazes the most the audience is good teamwork. How can the actors simultaneously make the proper decisions without any plot script and not turning the performance into a chaotic action? The secret is that the improvisation is not random. The actors practice a lot to perfect their possible role no matter which topic the audience gives them. As Gladwell insists, the spontaneity is rarely accidental [Gladwell, 2005: 52].

Of course, there are some occasions when our mental stimulus fails. It may be due to some biases we do not know we have as well as some external impetus that intervenes at a wrong time. This is what differs us from the computers. We have our principles; we follow morality; we depend on our cultural, social and educative background. We are autonomous in making our own decisions and predictions. The machine only knows how to respond to the impulses, yet, it still cannot do what is not encoded in its program. Imagine a massive (liner like Titanic was). In the lower compartment burst the big hole (again like Titanic had). The ship may drown if we do not immediately close the locks. Yet, we know that there are workers in there, who will undoubtedly sink if we do so. It will be a tough decision to make for a human because we can feel all the burden of responsibility for our choice. While the computer will close the locks without a hitch.

As we can see, even if we can do some actions on the "replay mood", the biggest decisions we always make slowly, calculating the options, comparing all the pros and cons, studying the possible consequences. Human remain conscious and think consciously (we are not counting rare mental instability cases) when something important is at stake. We can drive home unconsciously, focusing our mind on some other thoughts. For that, we need to know 1) how to drive the car, 2) the road, 3) some features of the car, etc. Yet, if there is some unexpected traffic situation, your mind direct your attention to the road or the car. At the same time, the machine operates according to the program without changing the "mind state."

\section{AI vs Human}

No doubt, the Artificial Intelligence (further in the text AI) is the number one topic in nowadays. Filmmaker scares us with the robots war; scientists surprise us with the new inventions. Indeed, we live in the world surrounded by the technical presence. To understand better what is AI, note that there are two kind of AI: (1) intelligent computers which can talk, think and behave like humans (it is the strong version that is not yet created); (2) computer 
systems able to work with Big Data and algorithms, perform the hard tasks and calculations, have the speech and face recognition, and so on. Not talking about robot Sophia, remember Siri, Alexa, Amazon, Tesla, PokemonGo. We have cars that drive themselves, we have phones that stop the videos when they lose our visual focus, and we have smart houses. All these are the good examples of, what is called, the weak AI models presented in our ordinary life.

These achievements are already significant, yet still far from being perfect. The computer that is told what it needs to do is not at all intelligent, is not it? More than 30 years now, scientists and engineers are developing the process of the machine (deep) learning. Their goal is to "bring the autonomy to the machine's world." Today the new-build technologies are capable of selfeducation using the reinforcement learning that is an unsupervised technique to learn by trial and error. However, does it mean that they are able to self-development in the full meaning of the word? As powerful as the recent technologies are considered (because of the fast connection to the enormous base of knowledge people collected), they still do not attain self-awareness. For the same reason, they not only cannot feel and understand their force but also use it in their favor. Nevertheless, let us imagine that such AI exists and it was created by Tesla. It is a brand new intelligent machine that cannot only decide for you the quickest way to get home, but also, the music and the smell correspondent to your mood. Which task do you think will be easier for such a New Tesla? The first one for sure. To do so AI must learn to predict the next human action. Thus, he should have in his database all the variants of possible ways home. Yet, to choose the music or the smell AI must understand human emotions. This is harder. It is not enough to read the facial expressions or to measure the pulse. Only empathy, the main characteristic that makes us human, can help the AI to do the right choice and not to play hit-or-miss.

To bring AI more human-like, scientists should not merely work on its look, but also on its "internal filling." I am talking about the replication of the structure of the human brain with all complicated nerve structure that covers the whole human body. The catch is that we still do not know everything about the human's brain function, so, we cannot totally input the "right duplicate" into the computer's program. Moreover, there is a thing called subconscious that has a strong influence on human behavior, decisions and desires, and we still know neither its location nor its employment. Despite all these gaps, the first design of the Neural Networking (just NN further in the text) impress. Deep learning, so important for the AI progress, uses large-scale NN, which consists of millions of simulated "neurons" structured in layers. This is an immature copy of the production of our brain, but the work is not finished yet.

Putting aside the technological news, I invite you now to talk more about the difference in human's decision-making process and one of the existent machines. Aristotle might be delighted to see his syllogism in work. AI paradigms include tools such as symbolic logic, fuzzy systems, evolutionary computing and probabilistic reasoning models in a limited way as they can. In programs made to solve the problems, the explicit logic and numerical calculations are provided. As Gloria Phillips-Wren and Lakhmi Jain say in their collective paper Artificial Intelligence for Decision Making, AI is usually used to solve the stressful decision problems; reducing information overload, enabling up-to-date information and the communication required for collaborative decisions; dealing with uncertainty; etc. [Phillips-Wren, 2006].

The most significant advantage of AI over a man is the quick multitasking. The average person cannot think two thoughts at the same time. Withal there is a limited amount of ideas people can hold in their head, and the searching process of the useful information can be long for the untrained minds. Herbert Simon, American economist whose primary interest was decision-making process, supposes that the real-life decisions, more or less 
contradictive as they are, are so due to human's limited rationality, or, how he named it, bounded rationality [Simon, 1972: 161-176] Scanning fast all the information it holds, a machine gives us a clear and unambiguous result. With no emotions involved, the AIbased program may aid the police officers to arrest without regret a person who, for instant, wais caught driving drunk for the n-time or help doctors prescribe the right treatment for a patient dying from cancer. This hybrid case-based reasoning that AI has is advantageous cooperation for humans because it is designed for storing vast amounts of information and rearranging it in .the exact combinations. This essential structural difference between machines programs and humans brains is the cause that makes us "think" so differently. Human brains learn slowly, compared to computers, and we hold only helpful or interesting information for us, while the electronic programs save everything, although it is meaningless to them. However, unlike computers, human are blest with the greatest gift — we can create something entirely new that had never existed before. Our brains, inspired by a good wine or classical music, can spontaneously combine the information in surprising new ways, recognizing original patterns and seeing the learned things in a completely different light. Machines can only recreate what they "know." They assemble the ideas without producing any original thoughts. They cannot invite a thing previously inexistent. No machine has not yet created something on their own, analyzing all the knowledge that they have access to via the Internet. Computers lack the imagination because it cannot be programming. However, it may be tried to be taught. Therefore, high hopes are placed on NN. The question is could the NN, even if they replicate a human brain, reach the "human capacities"? After all, NN is nothing but the software simulation made by ordinary programming computers, which works in an old-fashion way with serially connected logic gates, to behave as though they are billions of highly interconnected brain cells working in parallel.

Despite it fast development, the machines will not still be exactly like humans, because even if we generate an artificial brain that absolutely reflects the human brain functions (which is only a physical side), we should also teach the AI to use it in a human's way. Computers, strictly programmed as they are, always solve tasks using mathematical and logical tools what makes them excessively "rational" comparing to humans. Hedonistic, as we are, humans, in general, are searching for maximizing the satisfaction from their choices, while the rationality initiates us to work for a profit. The same fact explains why, for instance, many people are happy to leave the discussions with their opinion, their "own truth" instead of getting together to the bottom of the truth.

\section{Conclusions}

After all that was said, you can see that Aristotelian logic, no matter how good it is, is not applicable to the real-life thinking process that occurs in our head. As Nietzsche marked in his Human, All Too Human logic rests on assumptions that do not correspond to anything in the real world [Nietzsche, 1996: 22] Nevertheless, Aristotelian groundwork served as a reasonable basis and impetus for further development and improvement of logic. Due to Aristotle, the mathematical and computational logics, which are actively used today in the development of AI systems, had become possible. Cybernetics, robotics, programming owe their existence to this Ancient Greek philosopher.

Let us focus our attention on the "daughter" of Hanson robotics — robot Sophia. We can observe the masterpiece of a high-quality work of robotics for 2018. She looks like a real 
woman (at least her face does); she rapidly answers your replies; she understands (or better say, recognizes) jokes and can make one herself. Moreover, Sophia became the first robot, who gained the citizenship. Unfortunately, this is not enough to make the machines more humanlike. She still makes mistakes defining the body language, and cannot distinguish the sarcasm (sure, sometimes it may be far even for humans). Furthermore, even though she seems to catch and mimic human emotions, it does not mean she really feels them.

Despite all these Sophia's pluses and minuses, the fundamental difference (between her and us, humans) lays in the first principal used to create robots. We aim to make them rational, considering ourselves to be reasonable and logical. The problem is that there are too many factors that disorganize our analytical mind. This displace occurs because our cognitive processes have two systems: a fast one produced by the intuitive reactions, and a slow one that includes logic, reasoning, and analysis [Kahneman, 2011: 23]. Working together, one can replace the other influencing our behavior and decisions. For an illustration, imagine that you cracking on the hard integral or algorithm while your mom comes and ask you would you prefer more for dinner: lasagna or chicken soup. You quickly choose one option that first came into your mind as the most satisfying to stay focused on your primary activity.

It is this automatic mode that enrolls the habits we have, the most repetitive moves and decisions we make, etc. That is what can be called the repetitive mind. AI does not have it because the machines need to re-work, re-score, re-interpreted the given task, even if it was already done a minute before. For sure, they are doing this very quickly, but still, they cannot react immediately to the stimulus. (Maybe, they will do so in future. We will see) On the other hand, because of this capacity, humans usually fall under the influence of the cognitive biases in their judgments. The last ones enable faster solutions, so we follow them astray. David Kahneman and Amos Tversky showed the most common of cognitive biases in their paper Judgment under Uncertainty: Heuristics and Biases [Kahneman \& Tversky, 1974: 1124-1130].

The biological side of decision-making is a crucial viewpoint that we cannot ignore. Poorly identified processes always give bad results. For now, AI had learn how to operate the data need for the right predictions. Yet, we, human, use judgments made by our intuition, or feelings, or even mood, for example, for decision-making. AI differs from human, because of this disregard. We should accept that we are not creatures with "a powerful computer models" in our head. We are not as rational as we pretend to be. Stop lying to ourselves will be the best step the humanity should make if we want one day to create a better electronic version of ourselves. Then and only then we will be able to achieve the similarity between an AI model and human. Learning how the unconscious, cognitive biases, and emotions influence on our lives and decision-making process will bring us useful knowledge for further technical elaboration.

\section{References}

Aristotle. Organon, or logical treatises. London: George Bell \& sons, 1889. Vol. 1: 24b23-24. Damasio, Antonio. Descartes'error: emotion, reason, and the human brain. Avon Books. New York, 1995.

Gladwell, Malcolm. Blink. The power of thinking without thinking. Little Brown and Co., NY, and Boston, 2005.

Kahneman, Daniel. Thinking Fast and Slow. Farrar, Straus, and Giroux, 2011. 
Kahneman, Daniel and Tversky Amos. Judgment under Uncertainty: Heuristics and Biases. Science, New Series, Vol. 185, No. 4157, 1974: 1124-1131

King, Brian. Could a Robot be Conscious? (2018) Philosophy now. Electronic resources: https://philosophynow.org/issues/125/Could_a_Robot_be_Conscious

Klein, Gary. Sources of Power: How People Make Decisions. The MIT Press, Cambridge, Massachusetts, 1999.

Nietzsche Friedrich. Human, All Too Human. Cambridge University Press, 1996.

Phillips-Wren, Gloria, and Jain Lakshmi Chand. Artificial Intelligence for Decision Making. (2006) In: Gabrys B., Howlett R.J., Jain L.C. (eds) Knowledge-Based Intelligent Information and Engineering Systems. Lecture Notes in Computer Science, vol 4252. Springer, Berlin, Heidelberg

Simon, Herbert Alexander. A Behavioral Model of Rational Choice. The Quarterly Journal of Economics, Vol. 69, No. 1, 1955: 99-118.

Simon, Herbert Alexander. Theories of bounded rationality. (1972) In: C. B. McGuire and Roy Radner (eds.), Decision and Organization. North-Holland Publishing Company.

Thaler, Richard and Sunstein C. R. NUDGE: Improving Decisions About Health, Wealth, and Happiness. Yale University Press, New Haven \& London, 2008.

Tesla, Nicola. My Inventions. Electronic resources: http://www.teslasautobiography.com 